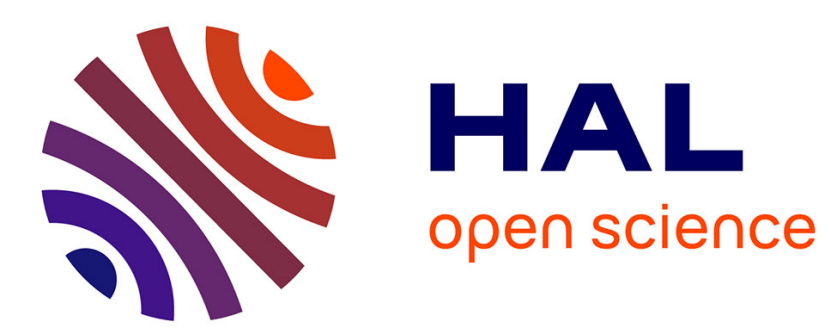

\title{
LIMITATIONS AND FURTHER DEVELOPMENT OF BEAM INJECTION METHODS. A SYNTHESIS
}

\author{
A. Jakubowicz
}

\section{To cite this version:}

A. Jakubowicz. LIMITATIONS AND FURTHER DEVELOPMENT OF BEAM INJECTION METHODS. A SYNTHESIS. Journal de Physique IV Proceedings, 1991, 01 (C6), pp.C6-325-C6-333. 10.1051/jp4:1991648 . jpa-00250734

\section{HAL Id: jpa-00250734 https://hal.science/jpa-00250734}

Submitted on 1 Jan 1991

HAL is a multi-disciplinary open access archive for the deposit and dissemination of scientific research documents, whether they are published or not. The documents may come from teaching and research institutions in France or abroad, or from public or private research centers.
L'archive ouverte pluridisciplinaire HAL, est destinée au dépôt et à la diffusion de documents scientifiques de niveau recherche, publiés ou non, émanant des établissements d'enseignement et de recherche français ou étrangers, des laboratoires publics ou privés. 


\title{
LIMITATIONS AND FURTHER DEVELOPMENT OF BEAM INJECTION METHODS. A SYNTHESIS
}

\section{A. JAKUBOWICZ}

IBM Research Division, Zurich Research Laboratory, Säumerstr. 4, CH-8803 Rüschlikon, Switzerland

\begin{abstract}
This paper gives a critical survey of the state of the art in the field of beam injection assessment of defects in semiconductors. It concentrates on recent developments, trends and current problems. A few statistical figures illustrate the development of this field in the past decade. The problems of spatial resolution and quantification of defect analyses are addressed. The paper closes with speculation as to further developments.
\end{abstract}

\section{Introduction}

Electronic micro-characterization of semiconductors is usually carried out by local excitation of the material. Commonly used are light and electron beams focused to very small dimensions. A large number of techniques, based on both kinds of excitation, has been developed in the past decade to study defects in semiconductors. Such spatially resolved techniques as EBIC, CL, OBIC, SDLTS, SEAM, PL and techniques related to them (for example IRBIC and QIRBIC (Cavallini, this workshop), or SMCTS (scanning minority carrier transient spectroscopy) have enabled us to investigate the homogeneity of semiconductor materials, to study the local properties of semiconductor devices and to understand the electrical properties of defects and their effect on the material/device on a microscopic scale. More recently, scanning tunneling microscopy (STM) has stimulated the development of novel injection techniques which have opened the chapter of electronic nano-characterization.

Electronic micro(nano)-charactcrization of semiconductors can be viewed in various respects. On the one hand there is an obvious interest in using these methods for fundamental research, as they give access to the electronic properties of individual defects, such as a single dislocation, grain boundary or even a single atom. On the other hand the sophistication of modern semiconductor electronics/optics and the ever smaller dimensions of devices make the application of these techniques inevitable for industrial purposes.

The number of papers on theory and applications of beam injection methods is immense. An extensive review of this field would necessitate a dedicated monograph, similar in character to that on SEM micro-characterization of semiconductors [1] or on cathodoluminescence of inorganic solids [2]. The intention of this paper is not to review this field, but rather to give a general overview of recent developments, to point out some important achievements, to discuss its limitations and to speculate on further developments.

\section{A Few Statistics}

The following statistical figures illustrate the development of beam injection techniques in the past decade. These figures have been elaborated using the INSPEC data base. Not all 
techniques have been included in these statistics; I selected only those which were frequently presented at this and previous workshops and thus appear to be representative for the field.

Figure 1 shows the total number of papers on EBIC, CL, OBIC, SEAM and SDLTS over the past ten years. Both papers discussing methods as well as those reporting their applications have been counted. The absolute numbers, however, should be interpreted with care, since a limited amount of key words was used for the literature search; these data should be treated only as approximate.

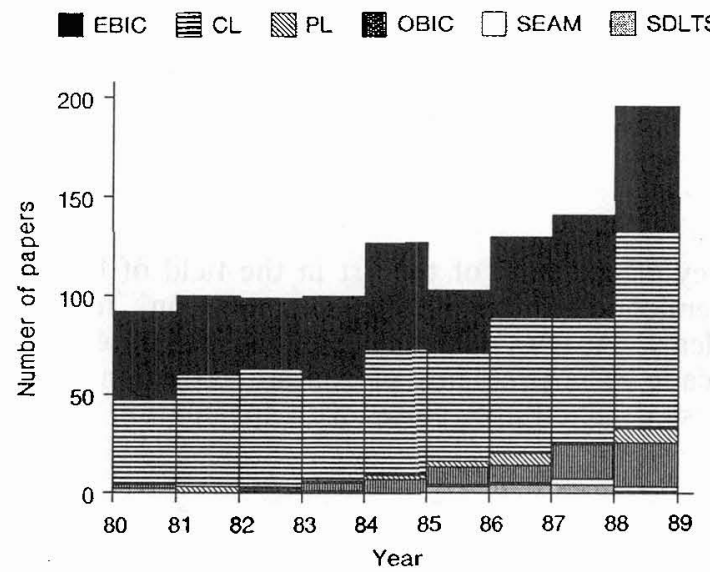

Fig. 1. The devclopment of beam injection techniques in the past decade.

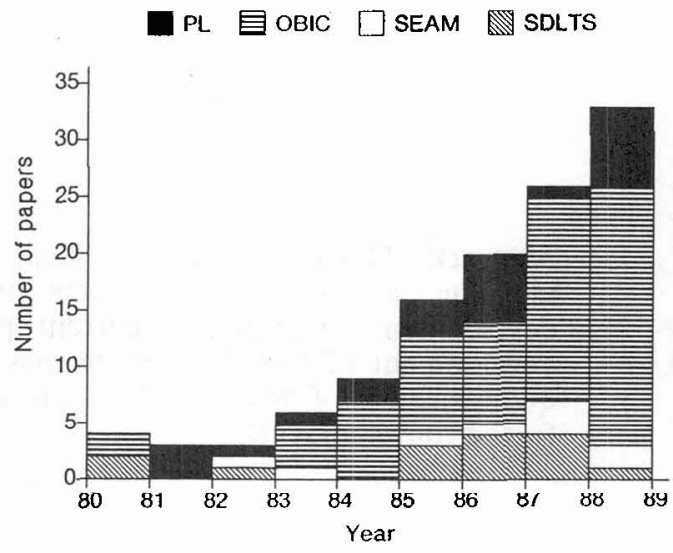

Fig. 2. The development of OBIC, spatially resolved $\mathrm{PL}, \mathrm{SDLTS}$ and SEAM.

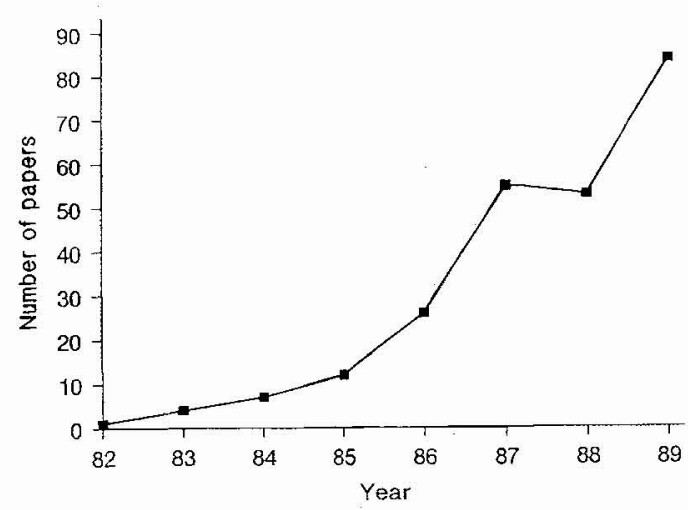

Fig. 3. The development of STM in the field of semiconductors.

In general one can say that a steady development characterized the past decade. There are significant differences among the individual techniques. EBIC and CL are predominant. Both techniques were the object of strong interest over the entire decade. The younger techniques have been exploited to a much lesser extent so far. The development of OBIC, PL (spatially resolved), SEAM and SDLTS is illustrated in Fig. 2 in an expanded scale. Of these four techniques $\mathrm{OBIC}$ has the highest growth rate. The fact that this histogram shows an increasing tendency allows us to be optimistic for the future.

Nano- and atomic-scale characterization experiences a dynamic development. Each year brings new techniques and new applications. Since many of them do not have yet well-defined names it is rather difficult to collect relevant statistical data. For comparison, however, the development of all kinds of STM applications in the field of semiconductors is shown in Fig. 3. 


\section{Spatial Resolution}

Spatial resolution is one of the most important parameters of any local technique, and therefore work on its improvement has continuously been of primary importance.

As far as the "older" techniques such as EBIC, OBIC, CL and PL are concerned, the factors that limit the spatial resolution in bulk material are believed to be well understood. First, when a beam interacts with a solid, a volume of finite dimensions is excited. Second, the final volume contributing to the signal can be increased by secondary effects, such as diffusion of carriers or, in the case of luminescence, the reabsorbed recombination radiation. In a system in which the resolution is not limited by these factors, it is determined by a characteristic screening length which depends on the concentration of free carriers and the density of charge in fixed positions (ionized atoms, trapped carriers). Most of the present efforts are aimed at improving the resolution in order to meet the demands of such applications as studies of heterointerfaces and low-dimensional systems. In these cases, questions arise which are related to the anisotropic transport of the injected carriers.

Possible ways to obtain a better resolution include reducing the beam-solid interaction volume, reducing the screening length by doping or temperature variation (emission of trapped carriers), time resolved measurements [3] and utilization of fields. Recently, in a CL study, Wada showed that a magnetic field can improve this parameter significantly (Lorentz force carrier confinement) [4]. Further investigations are needed here to understand better the effect of the magnetic field on $\mathrm{CL}$ and possibly on other signals $(\mathrm{EBIC}, \mathrm{OBIC}, . .$.$) in various$ systems in order to benefit from this technique.

In many practical situations today, optic and electron beams offer a similar spatial resolution. Although the electron beam does give a much smaller spot, its interaction with the semiconductor at typical SEM accelerating voltages increases the excited volume dramatically. On the other hand the scanning laser microscope has provided a spot size in the submicrometer range. The commercial availability of this instrument has made it an attractive partner of the SEM. It is worthwhile to mention that, in general, optical excitation has many advantages over electron beam excitation. The main advantage is that the former permits spectroscopic measurements to be performed by tuning the exciting wavelength.

In the past, the reported record values of the spatial resolution were in the range of a tenth of a micrometer [5]. Two major developments in recent years appear to be of particular importance. CL studies of quantum wells (QW) and scanning tunneling microscopy have moved the "traditional" micrometer limit of the spatial resolution of beam injection techniques into the nano-range. Nano-resolution in a $\mathrm{CL}$ study has already been reported by Christen and Bimberg at the first BIADS meeting (1988). In a two-dimensional system, such as a single QW, the emitted wavelength becomes sensitive to thickness variations of the order of one monolayer height. Thus by filtering out different wavelengths one can image these variations. Unfortunately this "simple" technique does not improve the lateral resolution. The latter, however, can be improved by one order of magnitude when low-voltage SEM is used. Recently, 600 angstroms has been reported by Warwick [6] for low-voltage CL measurements $(1 \mathrm{kV})$. This superior resolution was achieved by utilizing the high recombination velocity at the semiconductor surface in order to limit carrier diffusion. A similar value, 500 angstroms, is reported in this workshop by Wang, Steeds and Henini, who used TEM-CL to study cross-sectional specimens of GaAs/AlGaAs QW's.

There is an ongoing discussion on the achievable lateral and vertical resolution in SEAM [7]. It is understood that the resolution of this technique can be governed by the beam spot size, electron range, thermal diffusion and the minority carrier diffusion length [8]. This discussion will continue in the future in parallel with the discussion of the fundamental signal and contrast formation mechanisms.

In SDLTS the problem of spatial resolution is, at present, directly related to the sensitivity of the technique. Both the major difficulties and achievements lie on the instrumental side. The 
equipment recently developed offers a detection limit below $10^{-3}$ of the net doping concentration for a few microns of spatial resolution (Breitenstein and $R$ aith - this workshop). In practical terms this means that the sensitivity per scanning point can be better than 1000 atoms.

The invention of STM stimulated the development of new injection techniques operating on the nanometer scale. Such STM-related techniques as ballistic electron emission microscopy, luminescence, surface photovoltage and tunneling acoustic microscopy have allowed nano-scale imaging and characterization of defects. Until quite recently, the applicability of beam injection techniques was limited to characterization of extended defects, such as dislocations, grain boundaries, precipitates and large clusters of point defects. Today, atomically resolved carrier recombination [9] is "not surprising" any more, and selective injection into systems of reduced dimensionality, such as a single QW has become possible [10]. Whereas in the past, the characteristic distance related to transport of carriers, which was measurable by beam injection techniques, was the diffusion length, today, it has become possible to measure directly such quantities as the thermalization length of hot electrons [10]. It is not only the spatial resolution that makes STM an important injection technique. The low electron energies under which injection is carried out make it possible to perform spectroscopic investigations with a nanometer resolution [11]. Many other examples illustrating the capabilities of STM-based injection methods are available in the literature. A detailed review of this topic is, however, beyond the scope of this paper. From the point of view of defect analyses, nano- and micro-characterization are complementary fields.

\section{Trends, Current Problems}

Two basic trends of beam injection assessment of localized defects can be distinguished in the literature. These trends are particularly well pronounced for the most advanced techniques, i.e for $\mathrm{EBIC}$ and OBIC (recently also for $\mathrm{CL}$ and $\mathrm{PL}$ ). One trend is to treat the defect basically as a "black box" which is described by one or more phenomenological parameters characterizing its electronic behavior. The second trend attempts to give direct insight into the physical and chemical processes at defects.

The assessment of defects by an appropriate parameter, such as the recombination velocity at a grain boundary, constitutes an important part of applications of the beam injection techniques. Often, the goal of a defect analysis is a fast examination of the defect strength, without a detailed understanding of the physical processes. In device technology for instance, it may be of importance to know the "defect strength" expressed as a number, for example before and after a defect passivation technological process. For this part of applications of the beam injection methods there are in some cases well-developed thcoretical and experimental tools available, and ongoing efforts are being made to improve the accuracy of the models (c.g. Ref. 12) and experiments. EBIC, OBIC and luminescence can be used today in laboratories which characterize materials rather than work on the methods, although these techniques are still far from being routine. The latter is because in many practical cases the models use idealized assumptions, for example that a dislocation and a grain boundary are homogeneous objects, or that the defect is located in a homogeneous isotropic material. Also, the geometrical configurations considered are usually idealized as well. In the future there should probably be greater efforts to simulate realistic cases, including nonhomogeneous and anisotropic systems such as heterointerfaces and multilayer structures. This may only be possible by using numerical methods. First attempts in this direction have already been made. The problem of carrier injection in semiconductors with position-dependent band structure has been addressed numerically by Munnix and Bimberg [13]. A numerical model of the dislocation contrast problem (for EBIC applications) based on Monte Carlo simulation and solved by the finite difference method has been developed by Weber [14]. Czyzewski and Joy have performed a Monte Carlo simulation of CL and EBIC contrasts for isolated dislocations [15]. A numerical approach to the problem of multilayer structures has also been presented at this workshop by Aristov, Ushakov, Zaitsev and Yakimov. 
In high-resolution imaging the beam-solid interaction volume must be kept small. In this case, the object under investigation will always be very close to the surface. This problem requires more attention. The physical and chemical processes at and near surfaces can in general differ from those in the bulk. This can be of primary importance for a correct evaluation of results for any of the relevant techniques (c.g. Refs. 16 and 17). In this respect such models as that proposed for example by Farvacque and Sieber [18] to account for the effect of band bending near the surface are desirable. More work is necessary to investigate the various types of defect contrasts for EBIC, OBIC, CL and PL (and modifications of these methods). Most of the existing models treat only the "dark spot" case. At the present time work on other contrast types has a dominantly qualitative character; quantification is in its initial phase $[19,20]$.

Even the most exact and elegant phenomenological approach gives only limited understanding of the physical and chemical processes at defects. A good example of this is EBIC - until quite recently the most advanced quantitative technique. The limitations became cvident when a phenomenological approach was attempted in order to understand the measurements of the EBIC contrast of dislocations versus temperature [21-24]. In this case the turning point was the model of Wilshaw and Booker [25] - a good example of an elegant combination of the physical processes with a phenomenological quantity. Considering the fact that there is a great deal of experience in handling theoretically the standard EBIC and OBIC, it seems worthwhile to explore more intensively their applicability to spectroscopic measurements. Good examples of such techniques are IRBIC and QIRBIC (Cavallini, this workshop).

The fact that in recent years the work on physical models has been intensified can be viewed as a positive phenomenon (e.g. Ref. 26, and Tarento and Marfaing - this workshop).

One of the reasons that standard EBIC and OBIC today have the strongest and to a large extent experimentally tested quantitative aparatus is that by considering only two mechanisms, namely diffusion and recombination, which are also phenomenologically well understood, it was already possible to handle the problem of the defect contrast by a phenomenological theory, thus allowing a large number of experiments to be quantified for practically all semiconductors. The quantification of other techniques faces more difficulties. This is because the physical contrast mechanisms are not always fully understood or because several mechanisms act simultaneously with comparable contributions. A good example of the latter is SEAM, where acoustic, thermoelastic and electronic effects can contribute to the contrast. There is an ongoing discussion among SEAM experts on the signal and contrast mechanisms. The situation is quite complex. For example, it turns out that the signal and contrast origin is different for elemental and for (III-V) compound semiconductors (Ref. 7 and L.J. Balk, p. 425 in Ref. 1). Crucial experiments need to be carried out to separate the large number of possible mechanisms. There is an ongoing exploration of possible SEAM applications. Since there is no consistent SEAM theory for semiconductors, there is a lack of quantitative results, except for sporadic examples. Bresie, for instance, recently quantified the SEAM signal to determine the thermal conductivity of an implanted layer [27]. In the case of the thermal mechanism, theoretical expressions for the resultant stresses in a specimen with a free surface have been given in both the one- and three-dimensional cases $[8,27]$.

In the theory of spatially and spectrally resolved luminescence the difficulties stem from the necessity of taking into account the competitive nature of various recombination processes, including non-radiative recombination. Moreover, reflection of light, absorption, light interference, scattering and perturbations such as local stresses and electric fields must be considered. Independent of these difficulties, luminescence imaging today serves as a very efficient technique for the study of defects. Elegant examples of the potential of luminescence are imaging defects of single and multiple QW's at various wavelengths (monochromatic imaging) (c.g. Ref. 28) and electron temperature and lifetime mapping [29]. CL becomes increasingly attractive for studies of low-dimensional structures and devices based thereon. The CL technique has recently been reviewed in detail by Yacobi and Holt [2]. Unfortunately access to non-radiative processes by using CL and PL is only possible via measuring the losses of luminescence intensity (the "dark contrast"). Therefore SDLTS (and related techniques), which permits the local characterization of the nature of all types of deep point defect levels, is 
an important complementary spectroscopic technique. Since this technique is difficult from the experimental point of view, the number of its applications has increased only slowly. As far as the development of SDLTS is concerned, work concentrated on improving its sensitivity and on developing methods that allow the signals to be evaluated correctly. One of the difficulties in DLTS is that in many realistic cases the analyzed transients are not purcly exponential. Therefore part of the work in the past decade was focused on overcoming this problem. This work resulted in the development of appropriate procedures to evaluate non-exponential transients, such as the multi-exponential [30] and, more recently, numerical full-transient analysis methods [31]. The recent commercialization of SDLTS (Breitenstein and Raith, this workshop) allows us to conclude that many difficulties of experimental nature have been overcome, and leaves us with the hope that this technique will become accessible to a larger number of laboratories.

\section{Quantification - The "Ideal Software"}

The natural trend in the development of any technique is to quantify it, once the physical fundamentals have been well understood. Most of the beam injection techniques today serve as very efficient tools for qualitative studies, but in general their quantification is in the initial phase. How fast and in which direction quantification will develop in the future is currently but a subject of speculation. Several years ago Warwick proposed that the CL technique be quantified in order to be able to determine, for example, impurity concentrations from CL band intensities [32]. His idea was to develop a correction procedure for CL, analogous to that used routinely for X-rays, i.e. to the so-called ZAF correction procedure. Warwick called this future procedure MAS, which stands for mixed-level injection, absorption and surface recombination. Recently, Holt proposed in his review of $\mathrm{CL}[2 \overline{3}$ that reflection (and refraction) be added to this list and called the new set MARS. The concept to develop a versatile software which in the future would permit routine quantitative $C L$ measurements is very attractive, but faces many difficulties (the main limitation seems to be that an input of several experimental parameters varying from system to system is inevitable). The proposed correction software will, of course, grow each time a new physical process is taken into account. Therefore it is natural to ask how the most comprehensive set of corrections for quantitative $\mathrm{CL}$ should look. At the same time another question arises: why not develop a similar correction software for other local techniques? Lastly, the whole issue can be addressed in a more general way by asking the following question: how should the future quantitative software for defect characterization look?

Let us consider electron beam excitation in an SEM (the situation is similar for optical excitation). Electrons and holes generated by the electron beam can undergo several processes. They can be removed from a particular region of the sample by an internal or external field; they can be trapped or produce photons and phonons. These processes are the physical fundamentals of such techniques as EBIC, SDLTS, CL, and the acoustic mode. The interdependent character of these processes can be both advantageous and disadvantageous. On the one hand it may cause interpretation difficulties (the occurrence of one process can be the origin of artifacts in the evaluation of another one), but on the other hand it can be very helpful by providing missing information about the system and, indeed, it can be the starting point for the quantification of defect analyses. There are several problems to be solved in this respect. One of them stems from the fact that the defect-related contrast in any of the techniques is a complex multi-parameter function of the physical properties of the defect and the material. Another problem is that the injection of carriers may change the properties of the object under investigation significantly (both reversibly and irreversibly), thus making the analysis results very sensitive to the experimental conditions. One possible way to address these problems is similar to that of solving a set of equations with several unknowns, and it consists of a combined utilization of various techniques, provided the unknown parameters which determine the contrast are the same. In fact, such an approach is commonly practiced today by combining different techniques to analyze the same object. For example, combined applications of EBIC and SDLTS, CL and EBIC, SEAM and CL, and OBIC and PA (photoacoustics) have been frequently reported in recent literature [33-36]. Although such combined investigations today still have a predominantly qualitative character, recent developments allow 
one to be optimistic for the future. There are already several examples of a unified phenomenological description of various experiments (e.g. Refs. 37-39). Hergert has treated the EBIC, LBIC, CL, and PL signals in a unified way (this workshop). Zaitsev (this workshop) has considered the inverse problem [40] and showed that signals based on the registration of recombination events in excited media are described by the same equation. Preliminary quantitative experiments have demonstrated the strong potential of combined utilization of several techniques to characterize localized defects [41]. In this workshop, Schreiber and Hildebrandt derive from combined $E B I C / C L$ measurements both the relevant matrix parameters (optical absorption, diffusion length) and the defect depth (this workshop), while Eckstein and Habermeier (this workshop) show that combined EBIC/CL measurements permit the temperature dependence of the recombination activity of the defect to be determined even if the temperature dependence of the contrast is strongly influenced by that of other specimen parameters. As recent experiments have shown, in most cases, the same defect structures can be detected by several techniques. These various combinations of complementary methods (both stationary and time-resolved) should be treated quantitatively in the future not only phenomenologically but also using physical models. More experimental work in this direction is desirable as well.

Concluding this section, effective quantification appears to be via the simultaneous use of various beam injection techniques. Unfortunately, the present state of the individual techniques does not yet allow the development of a comprehensive software for the quantitative assessment of defects.

\section{Final Remarks and Summary}

The aim of this paper was not to review extensively the field of beam injection assessment of defects in semiconductors, but rather to give a critical survey of the state of art in this field. Hence, many valuable theoretical and experimental results have not been mentioned, and several techniques are not discussed. The number of techniques permitting local characterization of semiconductors is continuously growing. In this paper 1 referred only to those that comprise the main subject of this and the previous workshops. However, there are several other techniques which are somewhat outside of the mainstream of both workshops but which are nevertheless significant, as they can provide additional essential information about the material and defects. For example, micro-Raman spectroscopy (this workshop) can be used to determine the local structure and composition as well as local stresses and temperatures, and thus it can represent an additional "equation" in the complex system matrix/defect with several unknowns. "Voltage contrast" (there are various techniques based on this effect) is another example of a method which has been applied for many years to characterize local properties of semiconductor devices. In the field of electron and optical beam testing of devices several techniques have emerged in the past decade [42], concentrating on improving the resolution, sensitivity and the speed of testing.

Summarizing, in the past few years there has been significant progress made in the field of local characterization of semiconductors, but much remains to be done. Researchers in this field are continuously challenged by new questions regarding both fundamental research and rapidly developing semiconductor technology. Many of these questions reach the limits of the individual techniques. Therefore attention will probably have to be paid to utilizing several techniques simultaneously in measurements (both in the nanometer and micrometer range), and future work will have to be focused on numerical models in parallel to the analytical ones and on the development of new concepts.

\section{Acknowledgments}

I would like to thank D. Reichlin for her help in elaborating the statistical data, and L.-M. Schlosser, C. Jean-Richard and U. Bitterli from the Publications Department for their assistance in the final preparation of the manuscript. 


\section{References}

[1] "SEM Microcharacterization of Semiconductors", Ed.: D.B. Holt and D.C. Joy, Academic Press, London, 1989.

[2] "Cathodoluminescence Microscopy of Inorganic Solids", Ed.: B.G. Yacobi and D.B. Holt, Plenum Press, New York, 1990.

[3] J. Christen and D. Bimberg, Rev. Phys. Appl., 24, C6-85, 1989.

[4] K. Wada, A. Kozen, H. Fushimi and N. Inoue, Jpn. J. Appl. Phys., 27, L1952, 1988.

[5] A. Ourmazd, Contemp. Phys. 25, 251, 1984.

[6] C.A. Warwick, 7th Oxford Conference on Microscopy of Semiconducting Materials, Oxford, UK, 1991.

[7] L.J. Balk, M. Dominik and K. Kaufmann, Proceedings of SCANNING'91, Third International Conference on Confocal Microscopy and the Fourth International Conference on 3-D Image Processing in Microscopy, Atlantic City, New Jersey, USA, p.I-45, 1991.

[8] M. Qian and J.H. Cantrell, Mater. Sci. Eng., A122, 57, 1989.

[9] R.J. Hamers and K. Markert, Phys. Rev. Lett. 64, 1051, 1990.

[10] S.F. Alvarado, Ph. Renaud, D.L. Abraham, Ch. Schoenenberger, D.J. Arent and H.P. Meier, J. Vac. Sci.Technol. B, 9, 409, 1991.

[11] O. Albrektsen, D.J. Arent, H.P. Meier and H.W.M. Salemink, Appl. Phys. Lett. 57, $31,1990$.

[12] L. Pasemann, J. Appl. Phys. 69, 6387, 1991.

[13] S. Munnix and D. Bimberg, J. Appl. Phys. 64, 2505, 1988.

G. Weber, Ph.D. Thesis, Universitaet Koeln, 1990.

Z. Czyzewski and D.C. Joy, Scanning 12, 5, 1990.

S. Myhajlenko, R. Puechner, J.L. Edwards and D.B. Davito, 7th Oxford Conference on Microscopy of Semiconducting Materials, Oxford, UK, 1991.

S. Myhajlenko, Scanning Microscopy International, to be published.

J.L. Farvacque and B. Sieber, Rev. Phys. Appl. 24, C6-165, 1989.

C. Frigeri, Rev. Phys. Appl. 24, C6-184, 1989.

W. Seifert, H. Blumtritt and M. Kittler, Proceedings of the 6th Int'l Symp. on

"Structure and Properties of Dislocations in Semiconductors," Oxford, UK, p. 233, 1989.

[21] A. Ourmazd, P.R. Wilshaw and G.R. Booker, J. de Phys., Colloque C4, C4-289, 1983.

[22] L. Pasemann, Phys.Stat. Sol. A84, 133, 1984.

[23] A. Jakubowicz, J. Appl. Phys. 57, 1194, 1985.

[24] C. Donolato, J. Phys. 47, 171, 1986.

P.R. Wilshaw and G.R. Booker, Microscopy of Semiconducting Materials 1985, Conf. Series No.76, cis A.G. Cullis and D.B. Holt, p.329, 1985.

[28] J.W. Steeds, S.J. Bailey, J.N. Wang and C.W. Tu, in "Evaluation of Advanced Semiconductor Materials by Electron Microscopy", ed D. Cherns, Plenum Press, New York, NATO ASI Series B: Physics, Vol.203, p.127, 1989.

[29] Z.M. Wang, J. Windscheif, D.J. As and W. Jantz, Inst. Phys. Conf. Ser. 112, 191, 1990.

[30] J. Morimoto, T. Kida, Y. Miki and T. Miyakawa, Appl. Phys. A 39, 197, 1986.

T. Heiser, A. Mesli, E. Courcelle and P. Siffert, J. Appl. Phys. 64, 4031, 1988.

C.A. Warwick, Scanning Microscopy 1, 51, 1987.

O. Breitenstein and Ch. Eisenschmidt, Microelectronic Engineering 12, 171, 1990.

M. Eckstein, A. Jakubowicz, M. Bode and H.-U. Habermeier, Appl. Phys. Lett. 54, $2659,1989$.

B. Mendez and J. Piqueras, Inst. Phys. Conf. Ser. 100, 789, 1989.

T. Flohr and R. Helbig, IEEE Trans. Electron Devices 35, 1683, 1988.

L. Pasemann and W. Hergert, Ultramicroscopy 19, 15, 1986.

A. Jakubowicz, J. Appl. Phys. 59, 2205, 1986. 
T. Wilson and E.M. McCabe, phys. stat. sol. (a) 103, 107, 1987.

[40] C. Donolato, Inverse Problems 2, L31, 1986.

[41] M. Bode, A. Jakubowicz, H.-U. Habermeier, Proc. Conf. Defect Recognition and Image Processing II, Montrey 1987, ed E. Weber, Materials Science Monographs Ser.44, Elsevier, Amsterdam, p.155, 1987.

[42] J.-P. Collin, Rev. Phys. Appl. 24, C6-129, 1989. 